\title{
BUCKLING OF CYLINDRICAL SHELLS WITH SMALL CURVATURE*
}

\author{
BY \\ JOHN MALLET-PARET \\ Brown University, Providence
}

\begin{abstract}
We consider the bifurcation buckling of a rectangular plate with an imperfection of magnitude $\alpha$ under an applied lateral force of magnitude $\lambda$. The analysis allows the parameters $(\lambda, \alpha)$ to vary independently in a neighborhood of some $\left(\lambda_{0}, 0\right)$, and describes all buckled states of small magnitude. If the plate is represented by the domain $(0, \sqrt{ } 2) \times(0,1)$ in $R^{2}$, then the lateral force is applied to the edges $x=0, \sqrt{2}$, and the imperfection is a small vertical displacement of the form $z=(\alpha / 2) y^{2}(\sigma x+\tau)$, where $\sigma$ and $\tau$ are fixed. Roughly, then, the plate has a small curvature in the $y$-direction, of magnitude $\alpha(\sigma x+\tau)$.

The two cases $\sigma=0, \tau=1$ and $0<|\sigma|<<1, \tau=1$ are fully analyzed. In the first case, the plate has the form of a cylinder and possesses a high degree of symmetry. This results in a rather nongeneric bifurcation diagram. In the second case the symmetry has been broken, and a generic picture results.
\end{abstract}

1. The von Kármán equations. The von Kármán equations describe the buckling of a plate or shell under external forces. Consider, in fact, a rectangular plate described by the domain

$$
\Omega=(0, l) \times(0,1) \subseteq R^{2}
$$

in the $(x, y)$-plane. Assume that in the absence of external forces the plate is not perfectly flat, so it has a small imperfection which can be given as $z=\alpha w_{0}(x, y)$. Here $z$ is the displacement of the plate, $\alpha \in R$ is a small parameter and $w_{0}: \Omega \rightarrow R$ a known function. If now a lateral force of magnitude $\lambda$ is applied to the edges of the plate, the interaction of the resulting stress and the imperfection gives rise to a normal force, thus causing buckling of the plate. This is described by the equations

$$
\begin{aligned}
& \Delta^{2} f=-\frac{1}{2}[w, w]-\alpha\left[w, w_{0}\right] \\
& \Delta^{2} w=\left[w+\alpha w_{0}, f+\lambda F\right]
\end{aligned} \quad \text { in } \Omega,
$$

where

$$
[u, v]=u_{x x} v_{y y}+u_{y y} v_{x x}-2 u_{x y} v_{x y} .
$$

For a simply supported plate, the boundary conditions are

$$
f=0, \quad \Delta f=0, \quad w=0, \quad \Delta w=0 \quad \text { on } \quad \partial \Omega .
$$

* Received January 26, 1977. This research was supported in part the National Science Foundation under Grants GP-28931X3 and MPS $71-02923$ and in part by U.S. Army Research Office under Grants AROD DAH CO4-75-G-0077 and AROD AAG 29-76-6-0052. 
Here $F$ is the known Airy stress function of the applied forces and $f$ and $w$ are unknowns representing the additional stress and deflection caused by buckling. The final shape of the plate is thus

$$
z=\alpha w_{0}(x, y)+w(x, y) .
$$

See [14] for more details.

This and related problems have been studied by many people. A common choice for the applied force, which we shall take, is

$$
F(x, y)=-\frac{1}{2} y^{2},
$$

representing a compressive force applied to the edges $x=0, l$ acting in the $y$-direction. Knightly and Sather [12] and Matkowsky and Putnick [16] study the case where $\alpha=0$ and $\lambda$ is near an eigenvalue $\lambda_{0}$ of the linearized problem. Moreover, they set $l=\sqrt{ } 2$, which gives rise to a two-dimensional kernel for the linearized equations. There are also results in [11] where $\alpha$ is allowed to vary in a manner depending on $\lambda$. In [6], Chow, Hale and MalletParet considered a generic $w_{0}$, with $(w, \lambda, \alpha)$ varying independently in a full neighborhood of $\left(0, \lambda_{0}, 0\right)$, and List [15] studies the effect of letting also $l$ vary near 2 . In [13], Knightly and Sather study the buckling of a section of a cylinder curved in the $y$-direction; in our context this refers to $\alpha \neq 0$ fixed, $w_{0}(x, y)=\frac{1}{2} y^{2}$, and $\lambda$ varying near $\lambda_{0}$.

Here we study the buckling of a cylindrical section as the curvature $\alpha$ approaches zero; that is, $\alpha$ and $\lambda$ vary independently near 0 and $\lambda_{0}$. In view of the very specific choice of $w_{0}$, and the resulting high degree of symmetry, it turns out that the nature of the bifurcation is very nongeneric. We therefore also study the effect of superimposing an addition imperfection which destroys some of the symmetry and leads to a more generic picture. Physically, this corresponds to a cylinder with curvature in the $y$-direction, but varying slightly as a linear function of $x$.

As in [12], we may write (1.1), (1.2) as an operator equation in the Hilbert space

$$
X=\left\{u \in H^{2}(\Omega) \mid u=0 \text { on } \partial \Omega\right\},\langle u, v\rangle=\int_{\Omega}(\Delta u)(\Delta v) .
$$

Letting $\Delta^{-1}$ denote the inverse of the Laplacian, with zero Dirichlet data, set for $u, v \in X$

$$
\begin{gathered}
L u=\Delta^{-2}[u, F]=-\Delta^{-2} u_{x x}, \quad \Lambda u=\Delta^{-2}\left[u, w_{0}\right], \\
B(u, v)=\Delta^{-2}[u, v], \quad Q(u)=B(u, \Lambda u)+\frac{1}{2} \Lambda B(u, u), \\
C(u)=\frac{1}{2} B(u, B(u, u)), \quad p=-\Delta^{-2} w_{0 x x} .
\end{gathered}
$$

Then (1.1), (1.2) are equivalent to

$$
\left(I-\lambda L+\alpha^{2} \Lambda^{2}\right) w+\alpha Q(w)+C(w)=\alpha \lambda p .
$$

The first eigenvalue $\lambda_{0}$ of the compact self-adjoint operator $L$ is [12] $\lambda_{0}=9 \pi^{2} / 2$, and the kernel of $I-\lambda_{0} L$ is two-dimensional, with orthonormal eigenfunctions

$$
\phi_{j}(x, y)=\frac{2^{7 / 4}}{\pi^{2}\left(j^{2}+2\right)} \sin \frac{j \pi x}{\sqrt{ } 2} \sin \pi y, \quad j=1,2 .
$$

2. Plate with small cylindrical imperfection. In this section we consider the above problem with $l=\sqrt{2}$ and a small cylindrical imperfection given by

$$
w_{0}(x, y)=\frac{1}{2} y^{2} .
$$


Thus (1.3) becomes

$$
\left(I-\lambda L+\alpha^{2} L^{2}\right) w+\alpha Q(w)+C(w)=0 .
$$

As described above, we shall study the local bifurcation of $(2.2)$ for $(w, \lambda, \alpha)$ in a full neighborhood of $\left(0, \lambda_{0}, 0\right)$. The Lyapunov-Schmidt method (see [5] for details) immediately reduces $(2.2)$ to a finite-dimensional system of equations. First, decompose $w$ as follows:

$$
\begin{gathered}
w=P w+(I-P) w, \\
P w \stackrel{\text { def }}{=}\left\langle w, \phi_{1}\right\rangle \phi_{1}+\left\langle w, \phi_{2}\right\rangle \phi_{2} \\
\text { def } \\
\quad=u_{1} \phi_{1}+u_{2} \phi_{2} \in \operatorname{ker}\left(I-\lambda_{0} L\right), \\
(I-P) w=v \in\left[\operatorname{ker}\left(I-\lambda_{0} L\right)\right]^{\perp}
\end{gathered}
$$

where $u=\left(u_{1}, u_{2}\right) \in R^{2}$. Applying the projection $I-P$ to Eq. (2.2) gives rise to the auxiliary equation, which can be solved (by the implicit function theorem) for $v$ in terms of $(u, \lambda, \alpha) \in R^{2} \times R^{2}$; thus

$$
v=v^{*}\left(u_{1}, u_{2}, \lambda, \alpha\right)=O\left(|u|^{3}+|\alpha||u|^{2}\right)
$$

in a neighborhood of $\left(0, \lambda_{0}, 0\right)$. Now by substituting $v=v^{*}$ into (2.2) and applying the projection $P$, the bifurcation equations are obtained. Clearly they have the form

$$
f(u, \lambda, \alpha)=0, \quad f=\left(f_{1}, f_{2}\right) \in R^{2}
$$

where

$$
\begin{aligned}
f_{j}(u, \lambda, \alpha) & =\left(1-\frac{\lambda}{\lambda_{0}}+\frac{\alpha^{2}}{\lambda_{0}{ }^{2}}\right) u_{j}+\alpha\left\langle\phi_{j}, Q\left(u_{1} \phi_{1}+u_{2} \phi_{2}\right)\right\rangle \\
& +\left\langle\phi_{j}, C\left(u_{1} \phi_{1}+u_{2} \phi_{2}\right)\right\rangle+O\left(|u|^{5}+\alpha^{2}|u|^{3}\right)
\end{aligned}
$$

is analytic. The cubic polynomial in $(2.5)$ was calculated in $[6,12,16]$ and it was found that

$$
\begin{gathered}
\left\langle\phi_{1}, \mathrm{C}\left(u_{1} \phi_{1}+u_{2} \phi_{2}\right)\right\rangle=a u_{1}{ }^{3}+b u_{1} u_{2}{ }^{2}, \\
\left\langle\phi_{2}, \mathrm{C}\left(u_{1} \phi_{1}+u_{2} \phi_{2}\right)\right\rangle=b u_{1}{ }^{2} u_{2}+c u_{2}{ }^{3}, \\
a \simeq 3.945001 \times 10^{-4}, \quad b \simeq 5.007428 \times 10^{-4}, \quad c \simeq 1.623543 \times 10^{-4} .
\end{gathered}
$$

The quadratic term in (2.5) is given by Knightly and Sather [13] as

$$
\begin{aligned}
\left\langle\phi_{1}, Q\left(u_{1} \phi_{1}+u_{2} \phi_{2}\right)\right\rangle & =-5 k_{1} u_{1}{ }^{2}-3 k_{1} u_{2}{ }^{2},\left\langle\phi_{2}, Q\left(u_{1} \phi_{1}+u_{2} \phi_{2}\right)\right\rangle=-6 k_{1} u_{1} u_{2}, \\
k_{1} & =\frac{256}{(1215)(2)^{1 / 4} \pi^{6}} \simeq 1.842922 \times 10^{-4} .
\end{aligned}
$$

We shall defer a detailed analysis of the bifurcation equation (2.4) to Secs. 5-7.

Remark. The von Kármán equations here $((1.1),(1.2))$ are invariant under certain symmetries, and this is reflected in the bifurcation equation. Let $T: X \rightarrow X$ by $(T u)(x, y)=$ $u(\sqrt{ } 2-x, y)$. Then if we let $F(w, \lambda, \alpha)$ denote the left-hand side of the operator equation (2.2), clearly

$$
F(T w, \lambda, \alpha)=T F(w, \lambda, \alpha)
$$


Since $T$ also commutes with the eigenprojection $P$, it is easy to see that the bifurcation equations respect this symmetry in the sense that

$$
f(T u, \lambda, \alpha)=T f(u, \lambda, \alpha) .
$$

Here $T: R^{2} \rightarrow R^{2}$ is the induced transformation on the eigenspace and is given by $T\left(u_{1}, u_{2}\right)$ $=\left(u_{1},-u_{2}\right)$. Thus $f$ is an even function and $f_{2}$ an odd function of $u_{2}$. For a more general discussion of symmetries in bifurcation, see Sattinger [17, 18].

3. Cylindrical imperfection with varying curvature. Consider a modification of the problem in Sec. 2. Specifically, assume the imperfection in the plate has the form

$$
w_{0}(x, y)=\frac{1}{2} y^{2} \psi(x)
$$

where $\psi$ is a sufficiently smooth function of $x$. Thus the initial shape of the plate is curved in the $y$-direction, but this curvature $\alpha \psi(x)$ depends on $x$. In particular, this imperfection may exhibit less symmetry than (2.1) and one may expect that (3.1) will lead to a more generic bifurcation, as will be seen.

Certainly two differences between the equations involving the modified imperfection (3.1) and those of the previous section are clear:

1. In general $\Lambda \neq-L$ for the modified imperfection.

2. Also, in general $p=-\Delta^{-2} w_{0 x x} \neq 0$.

The situation in which the projection of $p$ onto $\operatorname{ker}\left(I-\lambda_{0} L\right)$ is non-zero (and satisfies some other generic conditions) was essentially treated in [6]. We shall therefore assume here that these projections vanish, and in fact, that $p$ itself is zero. Thus $\psi^{\prime \prime}(x) \equiv 0$ and we have for constants $\sigma, \tau$,

$$
w_{0}(x, y)=\frac{1}{2} y^{2}(\sigma x+\tau), \quad p=0 ;
$$

that is, the curvature of the plate in the $y$ direction varies linearly in $x$.

The effect of point 1 above is pronounced. Basically, for small $|\alpha|$, there is still a double eigenvalue at $\lambda=\lambda_{0}+\left(\alpha^{2} / \lambda_{0}\right)$ for the operator $I-\lambda L+\alpha^{2} L^{2}$. However, for small $|\alpha| \neq 0$, the double eigenvalue at $\lambda=\lambda_{0}$ in general splits into two nearby simple eigenvalues of the operator $I-\lambda L+\alpha^{2} \Lambda^{2}$. Although some symmetry-destroying phenomena also occur when the modified imperfection is considered, perhaps the most significant is this splitting of the eigenvalues.

One can place the problems considered here and that considered in [6] in the following perspective. The imperfection considered in [6] was essentially arbitrary, hence this situation is the most generic. The imperfection (2.1) of the previous section exhibits, as will be seen, a great deal of symmetry and is therefore the least generic. For the imperfection (3.1) some of this symmetry has been broken, and consequently this problem is intermediate between the other two. Of course here we are restricting our consideration to plates of length $l=\sqrt{ } 2$. The analysis of List [15], for example, does not fall into this classification.

Let us now derive the bifurcation equations for (1.3) with the modified imperfection (3.1). With the same decomposition (2.3) as before, the auxiliary variable $v$ satisfies the estimate $v^{*}=O\left(|u|^{3}+\alpha^{2}|u|\right)$. The bifurcation equations are then seen to be $f(u, \lambda, \alpha)=0$ where

$$
\begin{aligned}
f_{j}(u, \lambda, \alpha)= & \left(1-\frac{\lambda}{\lambda_{0}}\right) u_{j}+\alpha^{2}\left\langle\phi_{j}, u_{1} \Lambda^{2} \phi_{1}+u_{2} \Lambda^{2} \phi_{2}\right\rangle \\
& +\alpha\left\langle\phi_{j}, Q^{*}\left(u_{1} \phi_{1}+u_{2} \phi_{2}\right)\right\rangle+\left\langle\phi_{j}, C\left(u_{1} \phi_{1}+u_{2} \phi_{2}\right)\right\rangle+O\left(|u|^{5}+\alpha^{4}|u|\right) .
\end{aligned}
$$


The sharp on the quadratic term denotes that this term is different from the corresponding one in Sec. 2. The cubic terms are, however, the same. Note also the slight difference in the higher-order terms. The numerical calculation of the above terms, outlined in Sec. 8, yields

$$
\begin{aligned}
\left\langle\phi_{1}, u_{1} \Lambda^{2} \phi_{1}+u_{2} \Lambda^{2} \phi_{2}\right\rangle & =k_{2} u_{1}+k_{3} u_{2}, \\
\left\langle\phi_{2}, u_{1} \Lambda^{2} \phi_{1}+u_{2} \Lambda^{2} \phi_{2}\right\rangle & =k_{3} u_{1}+k_{4} u_{2}, \\
\left\langle\phi_{1}, Q^{\prime}\left(u_{1} \phi_{1}+u_{2} \phi_{2}\right)\right\rangle & =-\left(5 k_{1} u_{1}^{2}+3 k_{1} u_{2}^{2}\right)\left(\tau+\frac{\sigma}{2^{1 / 2}}\right)+\left(2 k_{7} u_{1} u_{2}\right) \sigma, \\
\left\langle\phi_{2}, Q^{\prime \prime}\left(u_{1} \phi_{1}+u_{2} \phi_{2}\right)\right\rangle & =-\left(6 k_{1} u_{1} u_{2}\right)\left(\tau+\frac{\sigma}{2^{1 / 2}}\right)+\left(k_{7} u_{1}^{2}+k_{8} u_{2}^{2}\right) \sigma
\end{aligned}
$$

where

$$
\begin{aligned}
k_{2}=\left(\frac{4}{81 \pi^{4}}\right) \tau^{2} & +\left(\frac{2^{5 / 2}}{81 \pi^{4}}\right) \tau \sigma+k_{5} \sigma^{2}, k_{3}=-\left(\frac{5(2)^{11 / 2}}{729 \pi^{6}}\right) \tau \sigma-\left(\frac{160}{729 \pi^{6}}\right) \sigma^{2}, \\
k_{4} & =\left(\frac{4}{81 \pi^{4}}\right) \tau^{2}+\left(\frac{2^{5 / 2}}{81 \pi^{4}}\right) \tau \sigma+k_{6} \sigma^{2}, \\
k_{5} & \simeq 3.440729 \times 10^{-4}, k_{6} \simeq 3.675239 \times 10^{-4}, \\
k_{7} & \simeq 1.939866 \times 10^{-4}, k_{8} \simeq 2.288281 \times 10^{-4} .
\end{aligned}
$$

4. Analysis of the abstract bifurcation equations. Recall the form of the bifurcation equations derived above:

$$
\begin{array}{r}
f^{*}(u, \lambda, \alpha)=\left(1-\frac{\lambda}{\lambda_{0}}+\frac{\alpha^{2}}{\lambda_{0}{ }^{2}}\right) u+\alpha Q^{*}(u)+C(u)+O\left(|u|^{5}+\alpha^{2}|u|^{3}\right), \\
f^{*}(u, \lambda, \alpha)=\left(1-\frac{\lambda}{\lambda_{0}}+\frac{\alpha^{2}}{\lambda_{0}^{2}}\right) u+\alpha^{2} M u+\alpha Q^{*}(u)+C(u)+\mathrm{O}\left(|u|^{5}+\alpha^{4}|u|\right) .
\end{array}
$$

An asterisk generally denotes terms arising from the equations considered in Sec. 2, while a sharp denotes those of Sec. 3. Here $u \in R^{2}, M$ is a symmetric $2 \times 2$ matrix, $Q^{*}$ and $Q^{*}$ are homogeneous quadratic polynomials and $C$ is a homogeneous cubic polynomial. For the remainder of this section these will be the only standing assumptions about $f^{*}$ and $f^{*}$, besides of course their smoothness. Using the approach of [6], we shall study zeros of Eqs. (4.1) and (4.2) near $u=0$, for parameter values $(\lambda, \alpha)$ varying independently in a full neighborhood of $(0,0)$. Then in Sec. 5 the particular bifurcation equations arising from the von Kármán problem will be analyzed in this framework.

First, to simplify notation, introduce a new parameter to replace $\lambda$ :

$$
\gamma=-1+\frac{\lambda}{\lambda_{0}}-\frac{\alpha^{2}}{\lambda_{0}^{2}}
$$

The following a priori estimate will justify the choice of scalings of the bifurcation equation.

Lemma 4.1. Assume $C$ satisfies the non-degeneracy

$$
C(u)=0 \text { implies } u=0 \text {. }
$$


Then all solutions $(u, \lambda, \alpha)$ near $(0,0,0)$ of $f^{*}=0$, or $f^{*}=0$, satisfy the estimate

$$
|u| \leq \text { const. }\left(|\gamma|^{1 / 2}+|\alpha|\right) \text {. }
$$

The easy proof this lemma follows that of Lemma 4.1 of [6].

Now restrict $(\gamma, \alpha)$ to one of the regions

$$
R_{1} \pm: \pm \gamma \geq K \alpha^{2}
$$

for a fixed $K>0$, and scale $(u, \gamma, \alpha)$ by making the replacements

$$
u \rightarrow|\gamma|^{1 / 2} u, \quad \alpha \rightarrow|\gamma|^{1 / 2} \alpha .
$$

The estimate (4.4) ensures no solutions will be lost in scaling. The bifurcation equations $f^{*}=0, f^{*}=0$ now become (after cancelling $|\gamma|^{3 / 2}$ )

$$
\begin{gathered}
\mp u+\alpha Q^{*}(u)+C(u)+O(|\gamma|)=0, \\
\left(\mp I+\alpha^{2} M\right) u+\alpha Q^{*}(u)+C(u)+O(|\gamma|)=0 .
\end{gathered}
$$

When $\alpha=\gamma=0$, if the resulting polynomial equations

$$
\mp u+C(u)=0
$$

have only simple zeros in the sense that the Jacobian determinant of (4.5) is non-zero there, then for large $K$, no bifurcation near $u=0$ occurs for parameters $(\gamma, \alpha)$ in the interior of $R_{1}^{ \pm}$. This is immediate from the implicit function theorem and implies that the number of small solutions remains constant in $R_{1}{ }^{-}$and in $R_{1}{ }^{+}$. The linearized stability of these solutions is determined by the Jacobian matrix of (4.5).

We now restrict the parameters to the region

$$
R_{2}:|\gamma| \leq K \alpha^{2}
$$

and analyze the solutions by means of the different scaling

$$
u \rightarrow \alpha u, \quad \gamma \rightarrow \alpha^{2} \gamma .
$$

The bifurcation equations now become

$$
\begin{gathered}
-\gamma u+Q^{*}(u)+C(u)+O\left(\alpha^{2}|u|^{3}\right)=0 \\
(-\gamma I+M) u+Q^{*}(u)+C(u)+O\left(\alpha^{2}|u|\right)=0 .
\end{gathered}
$$

Again this scaling is justified by Lemma 4.1. The scaled variables $u$ and $\gamma$ take values in some bounded set in $R^{2}$ and in the interval $[-K, K]$ respectively, while $\alpha$ lies in a small neighborhood of zero. By considering these values of $(u, \gamma, \alpha)$ together with the analysis for the regions $R_{1}{ }^{ \pm}$, the solutions in a full neighborhood of $(u, \gamma, \alpha)=(0,0,0)$ will have been described.

Define the Jacobian determinants

$$
\begin{aligned}
& \Delta_{1}^{*}(u, \gamma)=\operatorname{det}\left(-\gamma I+\frac{\partial Q^{*}}{\partial u}+\frac{\partial C}{\partial u}\right), \\
& \Delta_{1}{ }^{*}(u, \gamma)=\operatorname{det}\left(-\gamma I+M+\frac{\partial Q^{*}}{\partial u}+\frac{\partial C}{\partial u}\right)
\end{aligned}
$$

of the bifurcation equations at $\alpha=0$. (Below, the notation $\Delta_{j}, j=1$ or 2 , always refers to either $\Delta_{j}^{*}$ or $\Delta_{j}{ }^{*}$.) If $u^{0}$ is a solution of (4.6) or (4.7) when $\gamma=\gamma^{0}, \alpha=0$ and if $\Delta_{1} \neq 0$ there, 
then for $(\gamma, \alpha)$ near $\left(\gamma^{0}, 0\right)$ there is a unique solution $u$ near $u^{0}$ varying smoothly in $(\gamma, \alpha)$. Bifurcations in Eqs. (4.6), (4.7) thus occur near solutions $(u, \gamma, \alpha)=\left(u^{0}, \gamma^{0}, 0\right)$ at which $\Delta_{1}$ $=0$. If at that point the second-order determinant $\Delta_{2}$ described below is non-zero, then the bifurcation is particularly nice. More precisely, let

$$
\begin{aligned}
& \Delta_{2}^{*}(u, \gamma)=\left|\begin{array}{cc}
-\gamma I+\frac{\partial Q^{*}}{\partial u}+\frac{\partial C}{\partial u} & -u \\
\frac{\partial \Delta_{1}^{*}}{\partial u} & \frac{\partial \Delta_{1}^{*}}{\partial \gamma}
\end{array}\right| \\
& \Delta_{2}{ }^{*}(u, \gamma)=\left|\begin{array}{cc}
-\gamma I+M+\frac{\partial Q^{*}}{\partial u}+\frac{\partial C}{\partial u} & -u \\
\frac{\partial \Delta_{1}^{*}}{\partial u} & \frac{\partial \Delta_{1}^{*}}{\partial \gamma}
\end{array}\right|
\end{aligned}
$$

THEOREM 4.2. Assume (4.3) and let $u=u^{0}, \gamma=\gamma^{0}$ satisfy Eq. (4.6) or (4.7) when $\alpha=$ 0 . Suppose also $\Delta_{1}\left(u^{0}, \gamma^{0}\right)=0$ but $\Delta_{2}\left(u^{0}, \gamma^{0}\right) \neq 0$. Then there is a curve in the parameter space

$$
\gamma=g(\alpha), \quad g(0)=\gamma^{0}
$$

across which the number of solutions of (4.6) or (4.7) changes by two. These two solutions branch from a point $u=\phi(\alpha)$ where $\phi(0)=u^{0}$, and exist in the region $\gamma>g(\alpha)$ or $\gamma<g(\alpha)$ according to whether $\Delta_{2}\left(u^{0}, \gamma^{0}\right)>0$ or $\Delta_{2}\left(u^{0}, \gamma^{0}\right)<0$ respectively.

Proof. The proof of this result is similar to that of Theorem 4.1 of [6]. In particular, since $\Delta_{2} \neq 0$, one of the four entries of the Jacobian matrix at $\left(u^{0}, \gamma^{0}\right)$ is non-zero, say the $(1,1)$-entry. One may thus solve the first component of the bifurcation equation (4.6), or (4.7) for $u_{1}$ as a function of $u_{2}, \gamma$ and $\alpha$. This function is substituted into the second component, which thus reduces the problem to one equation with one unknown $u_{2}$. The condition $\Delta_{2} \neq 0$ also implies that this one-dimensional equation has the form

$$
h\left(u_{2}, \gamma, \alpha\right)=0
$$

where

$$
h\left(u_{2}, \gamma, \alpha\right)=(\text { const. })\left(u_{2}-u_{2}{ }^{0}\right)^{2}+(\text { const. })\left(\gamma-\gamma^{0}\right)+\text { h.o.t. }
$$

with non-zero constants and appropriate higher-order terms. One may then solve simultaneously

$$
h\left(u_{2}, \gamma, \alpha\right)=0, \quad \frac{\partial h}{\partial u_{2}}\left(u_{2}, \gamma, \alpha\right)=0
$$

as functions $u_{2}=\phi(\alpha), \gamma=g(\alpha)$. A nalysis as in [6] shows these functions have the required properties.

Remark. The trace of the Jacobian matrix at $\left(u^{0}, \gamma^{0}, 0\right)$ equals the (nonzero) eigenvalue of the linearization around this solution. Degree theory forces the other eigenvalue to be positive along one branch and negative along the other emanating from $u^{0}$. Thus the linearized stability of these solutions can be determined.

Remark. In unscaled coordinates the point from which the two solutions emanate has the form $u \sim \alpha u^{0}$. The corresponding curve in the parameter space has the form $\gamma \sim$ $\alpha^{2} \gamma^{0}$. 
Note that the point $(u, \gamma)=(0,0)$ in $(4.6)$ or $(u, \gamma)=\left(0, \gamma^{0}\right)$ where $\gamma^{0}$ is an eigenvalue of $M$ in (4.7) can never satisfy the conditions of Theorem 4.2, since at such points $\Delta_{2}$ vanishes. To study this situation one needs another a priori estimate, as well as another scaling; that is, the bifurcations of (4.6), (4.7) from $u=0$ must be analyzed. The results are given in the following theorems.

TheOREM 4.3. Suppose (4.3) holds and $Q^{*}$ satisfies

$$
Q^{*}(u)=0 \text { implies } u=0 .
$$

Assume also that

$$
-u+Q^{*}(u)=0 \text { implies } \operatorname{det}\left(-I+\frac{\partial Q^{*}}{\partial u}\right) \neq 0 .
$$

Then corresponding to each solution $u^{0}$ of (4.9) there is a solution

$$
u \sim \gamma u^{0}
$$

of (4.6) existing for $(\gamma, \alpha)$ near $(0,0)$. These are the only solutions in this neighborhood.

THEOREM 4.4. Suppose $M$ has distinct eigenvalues; let $\gamma^{0}$ be one of these, with $u^{0}$ the corresponding eigenvector. Then for $(\gamma, \alpha)$ near $\left(\gamma^{0}, 0\right)$, all the solutions of (4.7) near zero are the zero solution itself and a non-zero branch of the form

$$
\begin{aligned}
& u=\epsilon u^{0}+O\left(\epsilon^{2}\right),|\epsilon|<<1, \\
& \gamma=\gamma_{0}+O\left(\alpha^{2}\right)+\left[\frac{\left\langle u^{0}, Q^{*}\left(u^{0}\right)\right\rangle}{\left\langle u^{0}, u^{0}\right\rangle}+O\left(\alpha^{2}\right)\right] \epsilon+O\left(\epsilon^{2}\right) .
\end{aligned}
$$

In particular, if $\left\langle u^{0}, Q^{*}\left(u^{0}\right)\right\rangle \neq 0$ the branch may be written as

$$
u \sim\left(\gamma-\gamma^{0}+O\left(\alpha^{2}\right)\right)\left[\frac{\left\langle u^{0}, u^{0}\right\rangle}{\left\langle u^{0}, Q^{*}\left(u^{0}\right)\right\rangle}+O\left(\alpha^{2}\right)\right] u^{0} .
$$

Theorem 4.3 is due essentially to Knightly and Sather [13]. The proof involves obtaining an a priori estimate $|u| \leq$ (const.) $|\gamma|$ from (4.8), scaling $u \rightarrow \gamma u$, and applying the implicit function theorem to solve for $u$. Because the eigenvalues of $M$ are distinct, Theorem 4.4 is concerned with bifurcation from a simple eigenvalue. This situation is well known; see for example Crandall and Rabinowitz [7], or Chow, $\mathrm{Hale}$ and Mallet-Paret [5]. Note, incidentally, that the higher-order terms of (4.7) contain terms linear in $u$. Thus, the bifurcation occurs not necessarily exactly at an eigenvalue $\gamma^{0}$ of $M$, but rather at $\gamma^{0}+$ $O\left(\alpha^{2}\right)$.

The stability of these solutions is easily obtained. For (4.6), to the right $(\gamma>0)$ of the bifurcation, the eigenvalues of $-I+\left.\left(\partial Q^{*} / \partial u\right)\right|_{u^{0}}$ have the same signs as for the linearization of (4.6) around the solution, and the opposite signs for $\gamma<0$. For (4.7), there is one non-zero eigenvalue corresponding to the non-zero eigenvalue of $-\gamma^{\circ} I+M$, and another eigenvalue near zero. As is well known, the sign of this second eigenvalue along the non-zero solution is opposite to its sign on the zero solution.

5. Details of results for the von Kármán equations. In the previous section, various hypotheses were imposed on the terms of the bifurcation equations (4.1), (4.2), and several 
conclusions were drawn about the solutions of these equations as the parameters were varied. Here we shall apply these conclusions to the specific problems considered in Secs. 2 and 3. Let us first list the hypotheses mentioned above.

$$
\begin{gathered}
C(u)=0 \text { implies } u=0, \\
\pm u+C(u)=0 \quad \text { implies } \operatorname{det}\left( \pm I+\frac{\partial C}{\partial u}\right) \neq 0, \\
-\gamma u+Q^{*}(u)+C(u)=0 \text { implies } \Delta_{2}^{*}(u, \gamma) \neq 0, \\
\Delta_{1}^{*}(u, \gamma)=0, \quad u \neq 0 \\
(-\gamma I+M) u+Q^{*}(u)+C(u)=0 \quad \text { implies } \Delta_{2}^{*}(u, \gamma) \neq 0, \\
\Delta_{1}^{*}(u, \gamma)=0, \quad u \neq 0 \\
\quad Q^{*}(u)=0 \quad \text { implies } u=0, \\
-u+Q^{*}(u)=0 \quad \text { implies } \quad \operatorname{det} \quad\left(-I+\frac{\partial Q^{*}}{\partial u}\right) \neq 0,
\end{gathered}
$$

$M$ has distinct eigenvalues. If $u^{0}$ is of the eigenvectors then $\left\langle u^{0}, Q^{*}\left(u^{0}\right)\right\rangle \neq 0$.

Below are given the numerical values of the terms in the bifurcation equations:

$$
\begin{aligned}
& C(u)=\left(\begin{array}{c}
(3.945001) u_{1}{ }^{3}+(5.007428) u_{1} u_{2}{ }^{2} \\
(5.007428) u_{1}{ }^{2} u_{2}+(1.623543) u_{2}{ }^{3}
\end{array}\right) \times 10^{-4}, \\
& Q^{*}(u)=\left(\begin{array}{c}
-(9.214610) u_{1}{ }^{2}-(5.528766) u_{2}{ }^{2} \\
-(11.057532) u_{1} u_{2}
\end{array}\right) \times 10^{-4}, \\
& Q^{\prime \prime}(u)=\tau Q^{*}(u)+\sigma\left(\begin{array}{l}
-6.515713 u_{1}{ }^{2}+3.879732 u_{1} u_{2}-3.909428 u_{2}{ }^{2} \\
1.939866 u_{1}{ }^{2}-7.818856 u_{1} u_{2}+2.288281 u_{2}{ }^{2}
\end{array}\right) \times 10^{-4}, \\
& M=M_{1} \tau \sigma+M_{2} \sigma^{2} \\
& M_{1}=\left(\begin{array}{lr}
7.169527 & -3.228555 \\
-3.228555 & 7.169527
\end{array}\right) \times 10^{-4}, \\
& M_{2}=\left(\begin{array}{ll}
3.440729 & 2.282933 \\
2.282933 & 3.675239
\end{array}\right) \times 10^{-4} \text {. }
\end{aligned}
$$

It was observed in [6] that ( $\mathrm{H} 1)$ and ( $\mathrm{H} 2)$ hold. The unique solution $u=0$ of $u+C(u)$ $=0$ thus corresponds to the unique solution of $(4.1)$ or (4.2) in the parameter region $R_{1}{ }^{-}$. Because of the way in which $f^{*}$ and $f^{*}$ were defined, the two positive eigenvalues of the Jacobian $(\partial / \partial u)(u+C(u))=I$ imply this solution is stable (linearized stability). There are 
TABLE 1. Solutions of $-u+C(u)=0$ and signs of the eigenvalues of $-(-I+\partial C / \partial u)$.

\begin{tabular}{ccl}
\hline$u_{1}$ & $u_{2}$ & eigenvalues and stability \\
\hline 0 & 0 & + + unstable node \\
0 & \pm 78.482 & -- stable node \\
\pm 50.347 & 0 & -- stable node \\
\pm 42.574 & \pm 23.855 & -+ saddle (unstable) \\
\hline
\end{tabular}

nine solutions of $-u+C(u)=0$, and hence nine corresponding solutions of the bifurcation equations for $(\gamma, \alpha)$ in $R_{1}{ }^{+}$. Their stability is given in Table 1 ; see also $[6,12,16]$.

Calculations reveal that $\left(\mathrm{H}^{*}\right)$ does not hold. Table 2 lists the values $(u, \gamma)$ at which the scaled bifurcation equation (4.6) with $\alpha=0$ and its Jacobian determinant $\Delta_{1}{ }^{*}$ vanish. Because of the symmetry noted in Sec. 2, there is a point $(u, \gamma) \neq(0,0)$ at which $\Delta_{2}{ }^{*}$ also vanishes, so a special argument describing the behavior of solutions near this point is necessary and is given in Sec. 6. Briefly, for small $\alpha$ this point persists. As $\gamma$ increases past the critical value

$$
\gamma \simeq-4.1136 \times 10^{-4}+O(\alpha),
$$

two (saddle-point) solutions with $u_{2} \neq 0$ branch off the central solution with $u_{2} \equiv 0$, while this central solution changes from a saddle to a stable node. The form of the branching solutions is

$$
\begin{aligned}
& u_{1} \sim 1.7346 \times 10^{-4}, \\
& u_{2} \sim(65.978+O(\alpha))\left(\gamma+4.1136 \times 10^{-4}+O(\alpha)\right)^{1 / 2} .
\end{aligned}
$$

Near the other non-zero values in Table $2, \Delta_{2}{ }^{*} \neq 0$, so the form of the bifurcation is that described in Theorem 4.2.

It is easily seen that $\left(\mathrm{H} 4^{*}\right)$ holds. Also, ( $\left.\mathrm{H} 5^{*}\right)$, noted in Knightly and Sather [13], and the solutions of $-u+Q^{*}(u)=0$ along with their stability are given in Table 3 . Note that this stability is given by the eigenvalues of $I-\left(\partial Q^{*} / \partial u\right)$ and is valid to the right $(\gamma>0)$ of the bifurcation from $u=0$. To the left, the eigenvalues are of the opposite sign.

This analysis thus describes the number and form of the bifurcating solutions of (4.1) for $(\gamma, \alpha)$ in a full neighborhood of $(0,0)$. For small $\alpha \neq 0$, the bifurcation diagram in the $(\lambda, u)$ space is then similar to that given in Fig. 1. Moreover, by returning to the original unscaled parameters $(\lambda, \alpha)$, one is able to describe completely the number of solutions near $u=0$, as in Fig. 2. The number of solutions changes for parameter values on a curve of the form $\left(\lambda / \lambda_{0}\right)-1 \sim J \alpha^{2}$ for various constants $J$. Note that along the curve for $J=$

TABLE 2. Solutions of $-\gamma u+Q^{*}(u)+C(u)=0, \Delta_{1}{ }^{*}(u, \gamma)=0$, and trace of $-\left(-\gamma I+\partial Q^{*} / \partial u+\partial C / \partial u\right)$.

\begin{tabular}{cccccc}
\hline$u_{1}$ & $u_{2}$ & $\gamma \times 10^{4}$ & $\operatorname{trace} \times 10^{4}$ & $\Delta_{1}^{*} \times 10^{11}$ & $\begin{array}{c}\left(\gamma+1 / \lambda_{0}{ }^{2}\right) \times 10^{4} \\
=J \times 10^{4}\end{array}$ \\
\hline 1.1679 & 0 & -33.630 & .70322 & .53218 & -28.560 \\
1.7346 & 0 & -4.1136 & -7.7567 & 0 & .9560 \\
.97788 & .58852 & -5.4623 & -1.6169 & 2.8386 & -.2926 \\
.97788 & -.58852 & -5.4623 & -1.6169 & 2.8386 & -.2926 \\
0 & 0 & 0 & 0 & 0 & 5.0696 \\
\hline
\end{tabular}


TABLE 3. Solutions of $-u+Q^{*}(u)=0$ and signs of eigenvalues of $-\left(-I+\partial Q^{*} / \partial u\right)$.

\begin{tabular}{ccl}
\hline$u_{1}$ & $u_{2}$ & $\begin{array}{c}\text { eigenvalues and stability to right } \\
\text { of bifurcation }\end{array}$ \\
\hline 0 & 0 & ++ unstable node \\
-1085.2 & 0 & -- stable node \\
-904.36 & 522.13 & -+ saddle (unstable) \\
-904.36 & -522.13 & -+ saddle (unstable) \\
\hline
\end{tabular}

The signs of the eigenvalues are reversed to the left of the bifurcation.

$-.2926 \times 10^{-4}$ the number of solutions changes by four; this corresponds to two distinct bifurcations which are symmetrically situated about the $u_{1}$ axis in the $u$-plane. Also, we have included as a broken line the curve $1-\left(\lambda / \lambda_{0}\right)+\left(\alpha^{2} / \lambda_{0}{ }^{2}\right)=0$ for $J=\left(1 / \lambda_{0}{ }^{2}\right)=5.0696$ $\times 10^{-4}$, corresponding to the four curves of solutions branching from $u=0$.

Let us now compare the above analysis of $(4.1)$ with that for the modified problem (4.2). In particular, we shall consider $\tau=1$ and $\sigma \neq 0$ fixed and near zero. We claim that in this case hypotheses $\left(\mathrm{H} 3^{\prime \prime}\right)$ and $\left(\mathrm{H} 5^{\prime \prime}\right)$ hold. Thus by using Theorems 4.2 and 4.4 the bifurcation diagram and in particular the number of solutions can be obtained for $(\lambda, \alpha)$ in a full neighborhood of $(0,0)$.

One sees easily that $M$ has eigenvalues

$$
\lambda_{j}=\sigma \mu_{j}+O\left(\sigma^{2}\right), \quad j=1,2
$$

where $\mu_{1}, \mu_{2}$ are the distinct eigenvalues of $M_{1}$,

$$
\mu_{1} \simeq 3.9410 \times 10^{-4}, \quad \mu_{2} \simeq 10.398 \times 10^{-4} .
$$

The corresponding eigenvectors $v_{j}$ of $M$ are

$$
v_{1}=\operatorname{col}(1,1)+O(\sigma), \quad v_{2}=\operatorname{col}(1,-1)+O(\sigma) .
$$

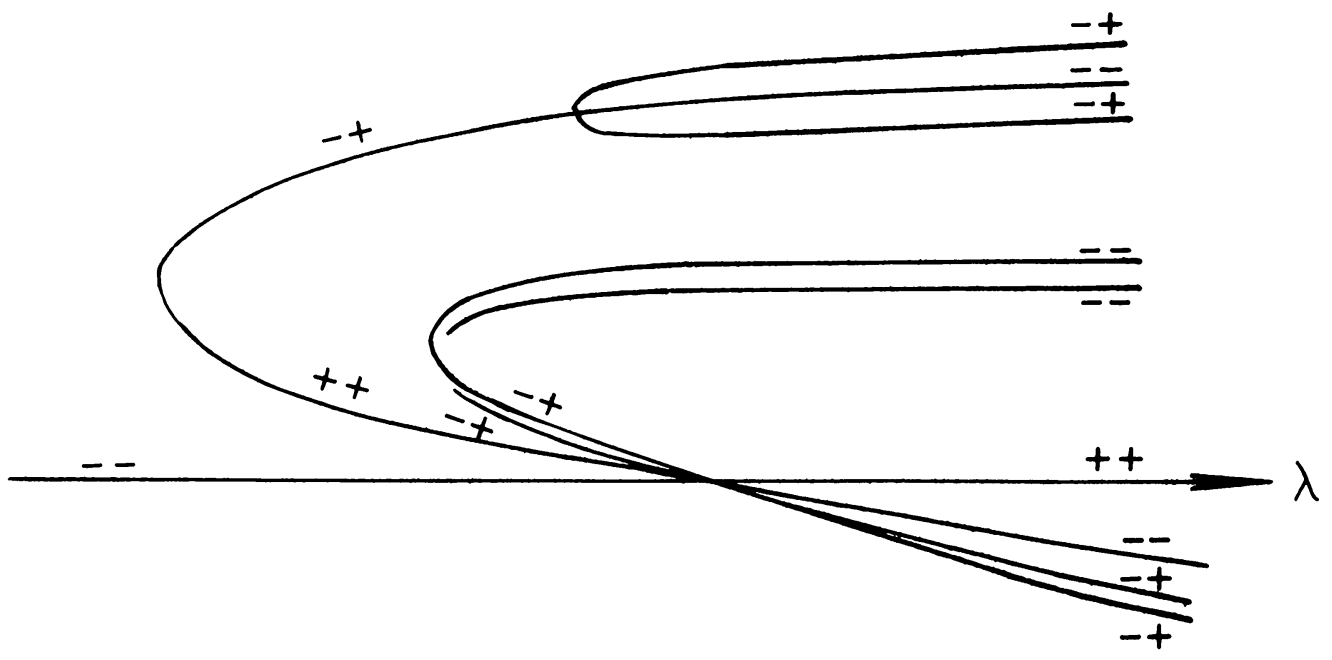

FIG. 1. Bifurcation diagram with $w_{0}(x, y)=\frac{1}{2} y^{2}$. 


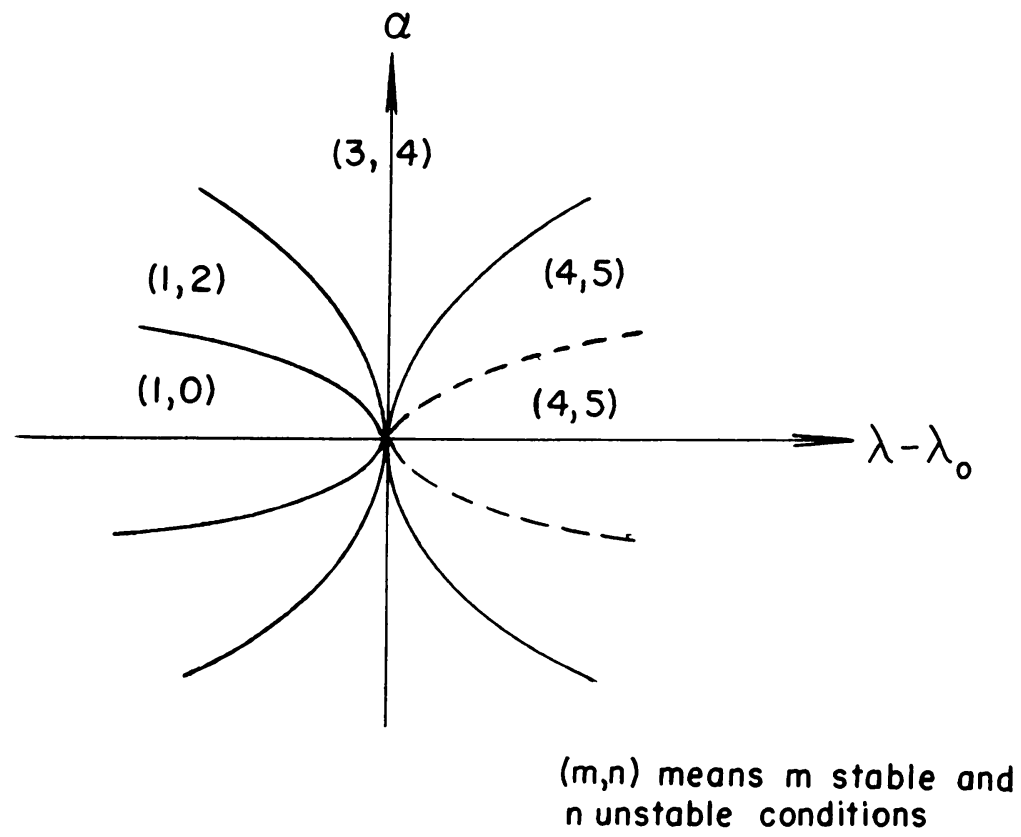

FIG. 2. Number of solutions with $w_{0}(x, y)=\frac{1}{2} y^{2}$.

Thus to check hypothesis $\left(\mathrm{H} 5^{*}\right)$, observe that

$$
\left\langle v^{1}, Q^{*}\left(v^{1}\right)\right\rangle=-25.801 \times 10^{-4}+O(\sigma), \quad\left\langle v^{2}, Q^{*}\left(v^{2}\right)\right\rangle=-25.801 \times 10^{-4}+O(\sigma) .
$$

Theorem 4.4 now applies, with the quantity

$$
\frac{\left\langle v^{j}, v^{j}\right\rangle}{\left\langle v^{j}, Q^{*}\left(v^{j}\right)\right\rangle}=-775.17+O(\sigma) .
$$

Checking $\left(\mathrm{H} 3^{*}\right)$ is more involved. It is clear from the implicit function theorem that, since $\left(\mathrm{H} 3^{*}\right)$ reduces to $\left(\mathrm{H} 3^{*}\right)$ when $\sigma=0$, those $(u, \gamma)$ in Table 2 for which $\Delta_{2}{ }^{*} \neq 0$ can be continued smoothly for small $|\sigma|$ as solutions to $\left(\mathrm{H} 3^{*}\right)$. As will be shown in Sec. 6, the point $\left(u_{1}, u_{2}, \gamma\right)=\left(1.7346,0,-4.1136 \times 10^{-4}\right)$ can also be continued for small $|\sigma|$, since we may regard the effect of the $\sigma$ terms in $\left(\mathrm{H}^{*}\right)$ as that of a forcing term in a onedimensional bifurcation problem.

Near $(u, \gamma)=(0,0)$, however, there appear several new solutions, basically due to the fact that the double eigenvalue $\gamma=0$ of $\left(\mathrm{H}^{*}\right)$ splits into two simple ones in (H3"). The analysis near this point depends on a scaling which is justified by the following lemma.

LEMMA 5.1. With $\left(\mathrm{H} 4^{*}\right)$ holding, and $M=M_{1} \sigma+O\left(\sigma^{2}\right)$, there is a neighborhood $\Re$ of $(u, \gamma)=(0,0)$ such that any solution of $\left(\mathrm{H} 3^{*}\right)$ in $\mathscr{N}$ for $|\sigma|$ sufficiently small in fact satisfies for some constant

$$
|u| \leq \text { const. }|\sigma|, \quad|\gamma| \leq \text { const. }|\sigma| .
$$

As with Lemma 4.1, the proof of Lemma 5.1 follows the corresponding one in [6] and so will not be given. By scaling $u=\sigma v, \gamma=\sigma \zeta$ one sees that 


$$
\begin{gathered}
(-\gamma I+M) u+Q^{*}(u)+C(u)=\left[\left(-\zeta I+M_{1}\right) v+Q^{*}(v)\right] \sigma^{2}+O\left(\sigma^{3}\right), \\
\Delta_{1}(u, \gamma)=\sigma^{2} \Delta_{1}{ }^{0}(v, \zeta)+O\left(\sigma^{3}\right), \quad \Delta_{2}(u, \gamma)=\sigma^{3} \Delta_{2}{ }^{0}(v, \zeta)+O\left(\sigma^{4}\right),
\end{gathered}
$$

where

$$
\begin{aligned}
\Delta_{1}{ }^{0}(v, \zeta) & =\operatorname{det}\left(-\zeta I+M_{1}+\frac{\partial Q^{*}}{\partial v}\right), \\
\Delta_{2}{ }^{0}(v, \zeta) & =\left|\begin{array}{cc}
-\zeta I+M_{1}+\frac{\partial Q^{*}}{\partial v} & -v \\
\frac{\partial \Delta_{1}{ }^{0}}{\partial v} & \frac{\partial \Delta_{1}{ }^{0}}{\partial \zeta}
\end{array}\right|
\end{aligned}
$$

Thus in order to verify $\left(\mathrm{H}^{*}\right)$ in the neighborhood $\mathscr{N}$, it is sufficient to find all solutions $(v$, $\zeta)$ with $v \neq 0$ of

$$
\left(-\zeta I+M_{1}\right) v+Q^{*}(v)=0, \quad \Delta_{1}{ }^{0}(v, \zeta)=0
$$

and verify that $\Delta_{2}{ }^{0}(v, \zeta) \neq 0$ at each. Since $\Delta_{2}{ }^{0}(v, \zeta)$ is the appropriate Jacobian with respect to $(v, \zeta)$, by the implicit function theorem such solutions can be continued for small $\sigma \neq 0$ as solutions of $\left(\mathrm{H}^{*}\right)$. The solutions of (5.2), as well as other relevant data, have been calculated and incorporated into Table 4 as the solutions for which $u=O(\sigma)$.

6. Details of results (continued). Here we shall examine more closely the behavior of solutions near the point $\left(\tilde{u}_{1}, \bar{u}_{2}, \bar{\gamma}\right)=\left(1.7346,0,-4.1136 \times 10^{-4}\right)$ where hypothesis $\left(\mathrm{H} 3^{*}\right)$ fails. It was noted in Sec. 2 that $f_{1}{ }^{*}$ is an even function and $f_{2}{ }^{*}$ an odd function of $u_{2}$. Thus the scaled bifurcation equation (4.6) can be written in the form

$$
F\left(u_{1}, u_{2}{ }^{2}, \gamma, \alpha\right)=0, \quad G\left(u_{1}, u_{2}{ }^{2}, \gamma, \alpha\right) u_{2}=0
$$

where

$$
\begin{gathered}
F\left(u_{1}, w, \gamma, \alpha\right)=-\gamma u_{1}-5 k_{1} u_{1}{ }^{2}-3 k_{1} w+a u_{1}{ }^{3}+b u_{1} w+O\left(\alpha^{2}\left|u_{1}\right|^{3}+\alpha^{2}\left|u_{1} w\right|+\alpha^{2} w^{2}\right), \\
G\left(u_{1}, w, \gamma, \alpha\right)=-\gamma-6 k_{1} u_{1}+b u_{1}{ }^{2}+c w+O\left(\alpha^{2} u_{1}{ }^{2}+\alpha^{2}|w|\right) .
\end{gathered}
$$

The solution $\bar{u}_{1}=k_{1} /(b-a), \bar{u}_{2}{ }^{2}=\bar{w}=0, \bar{\gamma}=(6 a-5 b) k_{1}{ }^{2} /(b-a)^{2}$ noted above is a simple zero of $F$ when $w=\alpha=0$; hence locally we can solve (6.1) uniquely for

$$
u_{1}=\mu\left(u_{2}^{2}, \gamma, \alpha\right) \text {. }
$$

In particular, $u_{1}=\mu(0, \gamma, \alpha), u_{2}=0$ represents a solution of both equations (6.1), (6.2). To obtain branches from this solution where $u_{2}=0$, let

$$
H(w, \gamma, \alpha)=G(\mu(w, \gamma, \alpha), w, \gamma, \alpha) .
$$

One may calculate that

$$
H(0, \bar{\gamma}, 0)=0, \quad \frac{\partial H}{\partial w}(0, \bar{\gamma}, 0) \neq 0, \quad \frac{\partial H}{\partial \gamma}(0, \bar{\gamma}, 0)=0,
$$

and, in fact, at $(0, \bar{\gamma}, 0)$,

$$
\begin{aligned}
-\frac{\partial H}{\partial \gamma}\left(\frac{\partial H}{\partial w}\right)^{-1} & =\frac{b-a}{2(3 a-2 b)^{2}+(5 b-7 a) c} \\
& \simeq 4353.2 .
\end{aligned}
$$


Thus one may solve $H=0$ for $w$ as a function of $\gamma$ and $\alpha$ to obtain

$$
w \sim(4353.2+O(\alpha))(\gamma-\bar{\gamma}+O(\alpha))
$$

which immediately yields (5.1) as required. Although further calculations reveal that the two branches (5.1) are saddles while the central solution $u_{1}=\mu(0, \gamma, \alpha)$ changes from a saddle to a stable node as $\gamma$ increases, these facts can also be deduced from arguments of Sec. 7.

To verify (H3") consider again the scaled bifurcation equations but at $\alpha=0$ and $0<$ $|\sigma| \ll 1$ :

$$
\begin{aligned}
F\left(u_{1}, u_{2}{ }^{2}, \gamma, 0\right)+\sigma F_{1}\left(u_{1}, u_{2}, \gamma\right)+O\left(\sigma^{2}\right) & =0, \\
G\left(u_{1}, u_{2}{ }^{2}, \gamma, 0\right) u_{2}+\sigma G_{1}\left(u_{1}, u_{2}, \gamma\right)+O\left(\sigma^{2}\right) & =0 .
\end{aligned}
$$

These equations are generally no longer symmetric. Since as before we may solve the first equation for $u_{1}$ and substitute this into the second, we may regard these equations as a one-dimensional bifurcation problem with a forcing parameter $\sigma$. The projection of this forcing term into the eigenspace (the $u_{2}$-axis) is

$$
G_{1}\left(\bar{u}_{1}, 0, \bar{\gamma}\right)=-\frac{5(2)^{1 / 2}}{729 \pi^{6}} \bar{u}_{1}+k_{7} \bar{u}_{1}^{2} \neq 0 .
$$

Arguments in [5] show that since this term is non-zero, there is for small $\sigma$ a locally unique solution of the bifurcation equations at which the Jacobian vanishes but the second-order Jacobian $\Delta_{2}$ " does not. Hence (H3") holds for $0<|\sigma|<<$.

7. The bifurcation diagram. The bifurcation diagram shows schematically how solutions $u$ behave as $\lambda$ is varied, and $\alpha=0$ is fixed and sufficiently small. To describe the diagram of the first problem, $f^{*}=0$, let us observe that the symmetry of $f^{*}$ implies the diagram must be symmetric with respect to reflection through the $\left(u_{1}, \lambda\right)$-plane. This imposes certain restrictions on how the solutions emanating from the various branches

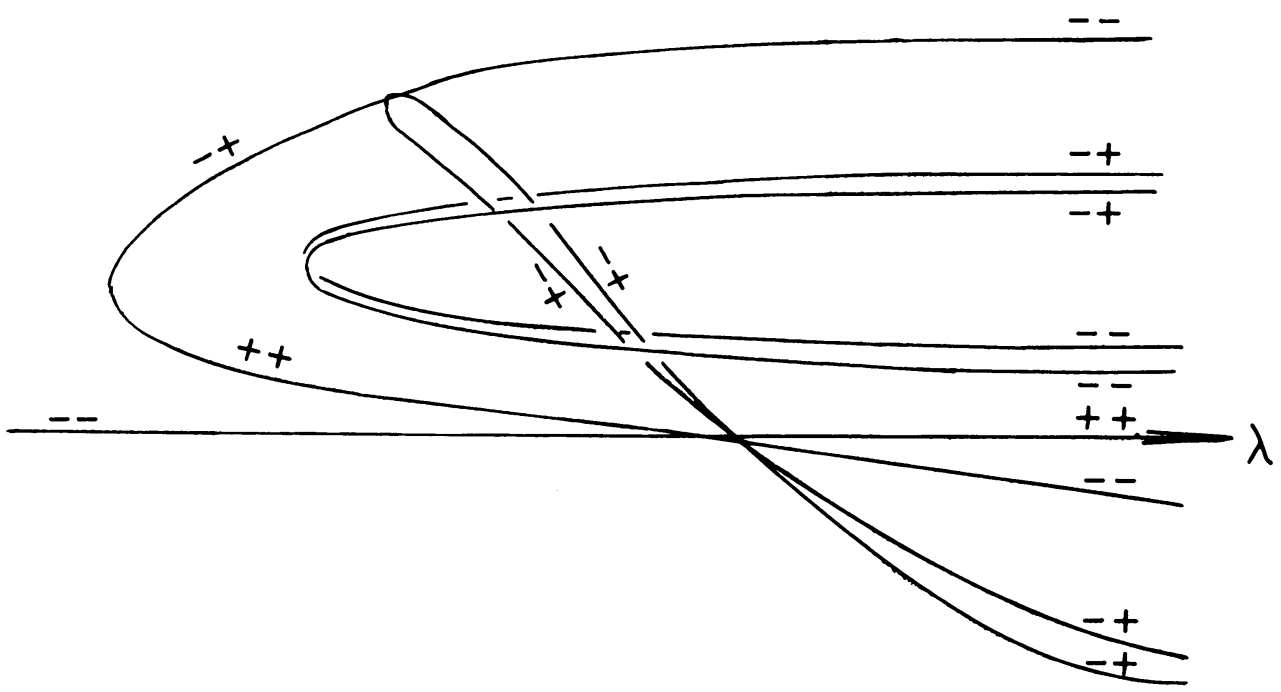

FIG. 3. Incorrect bifurcation diagram with $w_{0}(x, y)=\frac{1}{2} y^{2}$. 
may join together; for example, the pair of solutions branching to the right of $\gamma=-33.630$ $\times 10^{-4}$ (see Table 2) must both lie in the plane $u_{2}=0$ until they encounter another bifurcation. The stability of the various branches of course also imposes restrictions on how the solutions connect. Nevertheless, there are a priori two possible bifurcation diagrams (Figs. 1 and 3) consistent with Tables 1-3 and the above restrictions. The following argument shows, in fact, Fig. 1 is the correct one. If Fig. 3 were the case, there would be a $p=\left(u_{1}, u_{2}\right)$ such that

(a) $p$ would be a simple solution for some $\gamma$,

(b) $u_{2}=0$ would reach a maximum as $\gamma$ is varied.

This point would occur on one of the branches connecting the bifurcation at $\gamma=-4.1136$ $\times 10^{-4}$ with that at $\gamma=0$. Write the (scaled) bifurcation equations at $\alpha=0$ as

$$
\begin{gathered}
-\gamma u_{1}-5 k_{1} u_{1}{ }^{2}-3 k_{1} u_{2}{ }^{2}+a u_{1}{ }^{3}+b u_{1} u_{2}{ }^{2}=0, \\
-\gamma u_{2}-6 k_{1} u_{1} u_{2}+b u_{1}{ }^{2} u_{2}+c u_{2}{ }^{3}=0 .
\end{gathered}
$$

By regarding $u_{1}$ and $u_{2}$ as functions of $\gamma$ and differentiating $d u_{2} / d \gamma=0$ we obtain at $p$

$$
-u_{1}+\left(-\gamma-10 k_{1} u_{1}+3 a u_{1}^{2}+b u_{2}^{2}\right) \frac{d u_{1}}{d \gamma}=0, \quad-1+\left(-6 k_{1}+2 b u_{1}\right) \frac{d u_{1}}{d \gamma}=0
$$

and hence

$$
\gamma=(3 a-2 b) u_{1}^{2}+b u_{2}^{2}-4 k_{1} u_{1} .
$$

However, one sees from (7.2) that also $\gamma=b u_{1}{ }^{2}+c u_{2}{ }^{2}-6 k_{1} u_{1}$; thus

$$
(3 a-3 b) u_{1}{ }^{2}+(b-c) u_{2}^{2}+2 k_{1} u_{1}=0 .
$$

Finally, substitute the formula (7.3) for $\gamma$ into the first bifurcation equation (7.1), solve for $u_{2}^{2}$, and substitute this into Eq. (7.2) to yield

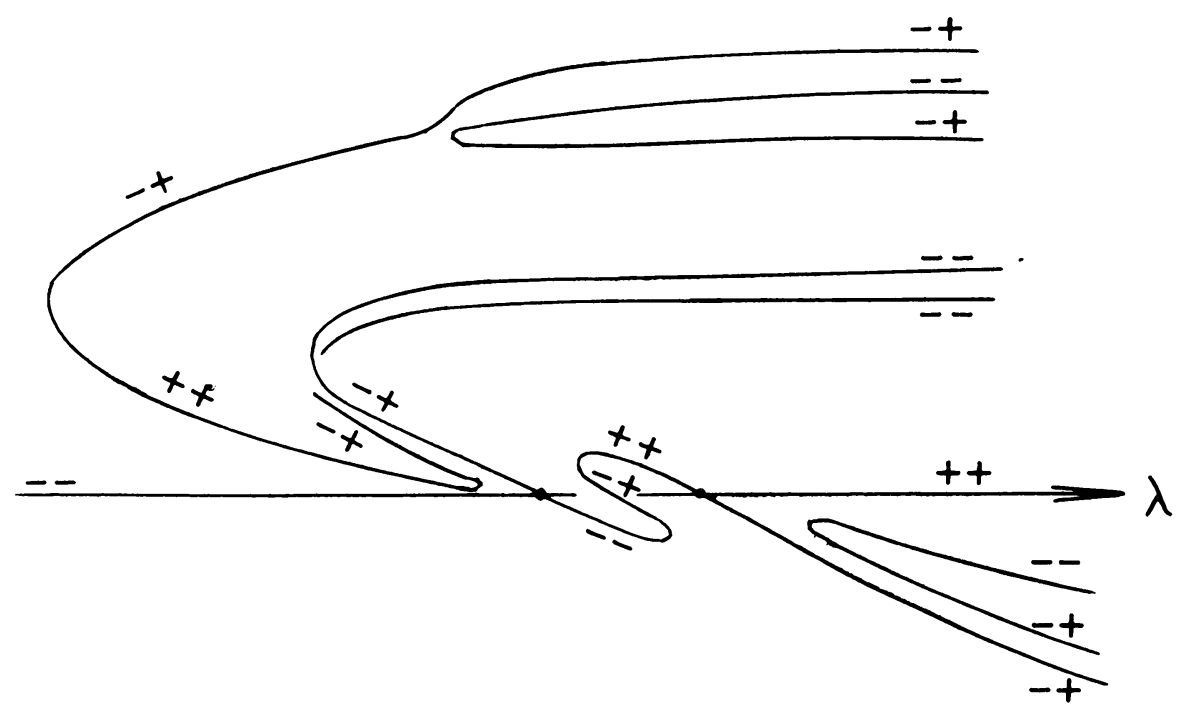

FIG. 4. Bifurcation diagram with $w_{0}(x, y)=\frac{1}{2} y^{2}(1+\sigma x), 0<|\sigma| \ll 1$. 


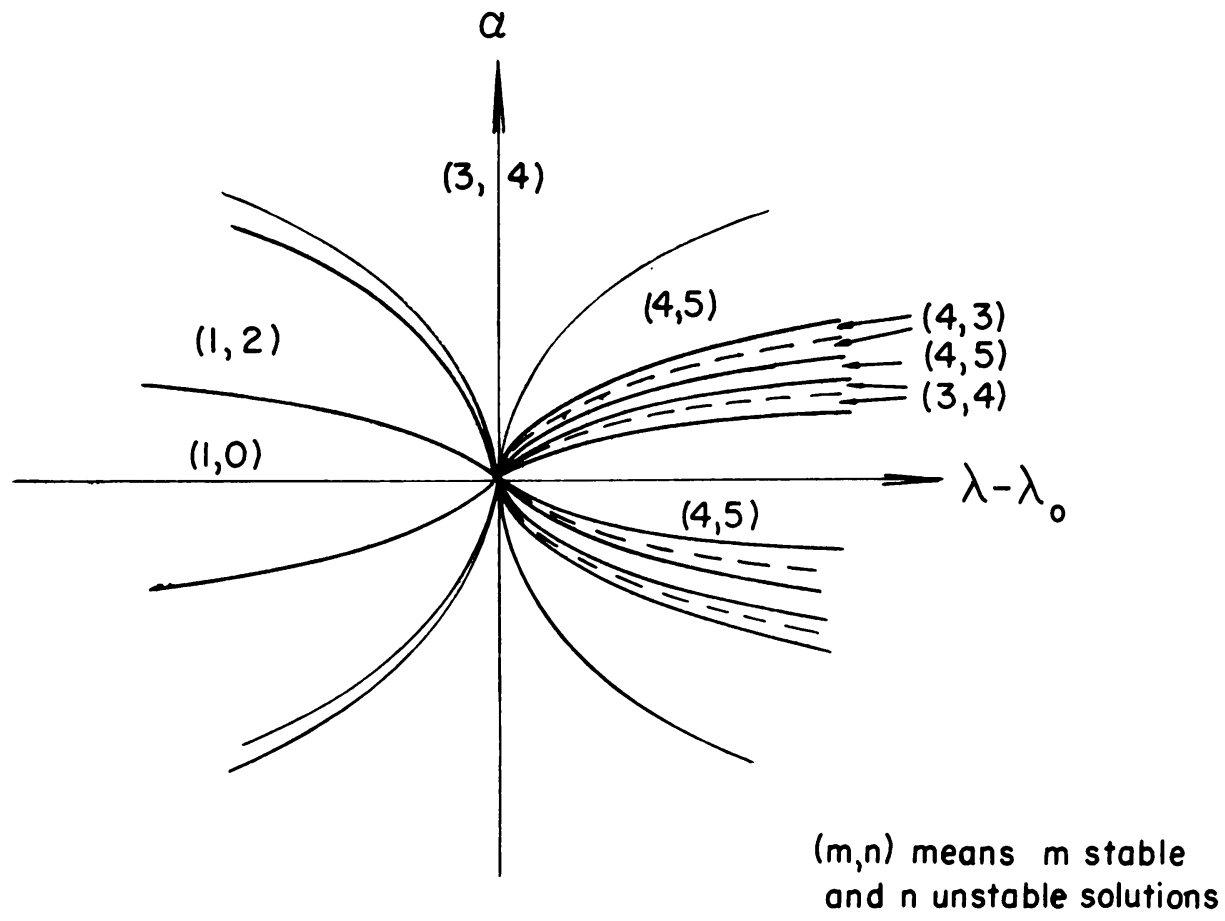

FIG. 5. Number of solutions with $w_{0}(x, y)=\frac{1}{2} y^{2}(1, \sigma x), 0<|\sigma|<<1$.

$$
2(a-b)(b-c) u_{1}{ }^{2}+(10 b-c-9 a) k_{1} u_{1}-6{k_{1}}^{2}=0 .
$$

Calculation of the discriminant of (7.4) shows there are no real solutions. Thus, Fig. 1 represents the correct bifurcation diagram.

Fig. 4 illustrates the bifurcation diagram for the problem $f^{*}=0$. Note that in Table 4 there are two occurrences of $\gamma=-5.4623 \times 10^{-4}+O(\sigma)$. One would expect that these values would be different for $0<|\sigma|<<1$; however, we have not calculated this here. The relative position of these two branches is thus not clear. Fig. 5 gives the number of solutions of $f^{*}=0$ as a function of the parameters $(\lambda, \alpha)$.

8. Remarks on the calculation of coefficients. We make some observations on the numerical calculation of the coefficients of the bifurcation equations. Note first that the operator equation (1.3) is equivalent [11] to the variational problem of finding critical points of

$$
J(w)=\frac{1}{2}\left\langle w,\left(I-\lambda L+\alpha^{2} \Lambda^{2}\right) w\right\rangle+\frac{\alpha}{3}\langle w, Q(w)\rangle+\frac{1}{4}\langle w, C(w)\rangle-\alpha \lambda\langle w, p\rangle .
$$

In particular, this implies that the functions $f^{*}$ and $f^{*}$ are actually gradients of a scalarvalued function; thus $M$ is a symmetric matrix, and restrictions are placed on the coefficients of $Q^{*}$ in (4.2).

Note also that when $\sigma=-2^{1 / 2} \tau$, the imperfection $w_{0}$ obeys the symmetry $T w_{0}=-w_{0}$. Since we also have $T \phi_{1}=\phi_{1}$ and $T \phi_{2}=-\phi_{2}$, it follows that $k_{3}=0$, and the only terms left to determine are $k_{5}, k_{6}, k_{7}$ and $k_{8}$ of (3.2). A lengthy calculation yields 


$$
\begin{aligned}
& k_{4+j}=\frac{2}{81 \pi^{4}}+\frac{128}{9 \pi^{9}} \sum_{\substack{p+j=\text { odd } \\
p \geq 1}} \frac{p^{2}\left(p^{2}+j^{2}\right)^{2}}{\left(p^{2}-j^{2}\right)^{4}\left(p^{2}+2\right)^{2}} \\
& +\frac{2048}{9 \pi^{8}} \sum_{q=2}^{\infty} \sum_{\substack{p+j=\text { odd } \\
p \geq 1}} \frac{p^{2} q^{2}}{\left(p^{2}-j^{2}\right)^{2}\left(p^{2}+2 q^{2}\right)^{2}\left(q^{2}-1\right)^{2}}
\end{aligned}
$$

for $j=1,2$, and

$$
\begin{aligned}
k_{6+j}= & \frac{512(2)^{1 / 4}}{27 \pi^{8}}\left[\sum_{\substack{p=\text { odd } \\
p \geq 1}} \frac{p\left(p^{2}+4\right)}{\left(p^{2}-4\right)^{2}\left(p^{2}+2\right)^{2}}\left(\frac{p}{4 j^{2}-p^{2}}+\frac{1}{3 p}\right)\right. \\
& \left.+\sum_{\substack{q=\text { odd } \\
q \geq 3}} \sum_{\substack{p=\text { odd } \\
p \geq 1}} \frac{4 p q}{\left(p^{2}-4\right)\left(q^{2}-1\right)\left(p^{2}+2 q^{2}\right)^{2}}\left(\frac{p}{\left(4 j^{2}-p^{2}\right) q}+\frac{q}{\left(4-q^{2}\right) p}\right)\right] \\
& +\frac{2048(2)^{1 / 4}}{27 \pi^{8}}\left[\sum_{\substack{j=\text { odd } \\
p \geq 1}} \frac{p^{2}\left(p^{2}+j^{2}\right)\left(p^{2}+j^{2}+4\right)}{3\left(p^{2}-j^{2}\right)^{2}\left(p^{2}+2\right)^{2}\left[p^{2}-(j-2)^{2}\right]\left[(j+2)^{2}-p^{2}\right]}\right. \\
& \left.+\sum_{\substack{q=\text { odd } \\
q \geq 3}} \sum_{\substack{p+j=\text { odd } \\
p \geq 1}} \frac{4 p^{2} q\left(2 p^{2}-p^{2} q^{2}+j^{2} q^{2}+4 q^{2}\right)}{\left.\left(p^{2}-j^{2}\right)\left(q^{2}-1\right)\left(p^{2}+2 q^{2}\right)^{2} q\left(4-q^{2}\right)\left[p^{2}-(j-2)^{2}\right][j+2)^{2}-p^{2}\right]}\right]
\end{aligned}
$$

for $j=1,2$.

\section{REFERENCES}

[1] L. Bauer and E. Reiss, Nonlinear buckling of rectangular plates, SIAM J. 13, 603-626 (1965)

[2] L. Bauer, E. Reiss and H. Keller, Multiple eigenvalues lead to secondary bifurcations, SIAM Review 17 , $101-122(1975)$

[3] M. Berger, On von Kármán's equations and the buckling of a thin elastic plate: I. The clamped plate, Comm. Pure Appl. Math. 20, 687-719 (1967)

[4] M. Berger and P. Fife, On von Kármán's equations and the buckling of a thin elastic plate: II. Plate with general edge conditions, Comm. Pure Appl. Math. 21, 227-241 (1968)

[5] S. N. Chow, J. Hale and J. Mallet-Paret, Applications of generic bifurcation I, Arch. Rat. Mech. Anal. 59. 159-188 (1975)

[6] S. N. Chow, J. Hale and J. Mallet-Paret, Applications of generic bifurcation II, Arch. Rat. Mech. Anal. 62, 209-235 (1975)

[7] M. G. Crandall and P. H. Rabinowitz, Bifurcation from simple eigenvalues, J. Func. Anal. 8, 321-340 (1971)

[8] J. P. Keener, Perturbed bifurcation theory at multiple eigenvalues, Arch. Rat. Mech. Anal. 56, 348-366 (1974)

[9] J. P. Keener, Secondary bifurcation and perturbed bifurcation theory, preprint.

[10] J. Keener and H. Keller, Perturbed bifurcation theory, Arch. Rat. Mech. Anal. 50, 159-175 (1973)

[11] G. H. Knightly and D. Sather, On nonuniqueness of solutions of the von Kármán equations, Arch. Rat. Mech. Anal. 36, 65-78 (1970)

[12] G. H. Knightly and D. Sather, Nonlinear buckled states of rectangular plates, Arch. Rat. Mech. Anal. 54, 356-372 (1974)

[13] G. H. Knightly, Some mathematical problems from plate and shell theory, in Proceedings of the Michigan State University Conference, Nonlinear Functional Analysis and Differential Equations, Marcel Dekker, Inc., New York, 1976

[14] L. D. Landau and E. M. Lifshitz, Theory of elasticity, Pergamon Press, 1970

[15] S. E. List, Generic bifurcation with application to the von Kármán equations, thesis, Brown University, Providence, R. I., 1976

[16] B. Matkowsky and L. Putnick, Multiple buckled states of rectangular plates, Int. J. Nonlinear Mech. 9. 89-103 (1974)

[17] D. H. Sattinger, Group representation theory and branch points of nonlinear functional equations, SIAM J. Math. Anal. 8, 179-201 (1977)

[18] D. H. Sattinger, Group representation theory, bifurcation theory and pattern formation, preprint. 